\title{
Tolerant reasoning: nontransitive or nonmonotonic?
}

\author{
Pablo Cobreros ${ }^{1}$. Paul Egré ${ }^{2}$ - Dave Ripley ${ }^{3}$. \\ Robert van Rooij ${ }^{4}$ (iD
}

Received: 3 March 2016 / Accepted: 30 September 2017 / Published online: 22 October 2017 (C) The Author(s) 2017

\begin{abstract}
The principle of tolerance characteristic of vague predicates is sometimes presented as a soft rule, namely as a default which we can use in ordinary reasoning, but which requires care in order to avoid paradoxes. We focus on two ways in which the tolerance principle can be modeled in that spirit, using special consequence relations. The first approach relates tolerant reasoning to nontransitive reasoning; the second relates tolerant reasoning to nonmonotonic reasoning. We compare the two
\end{abstract}

\footnotetext{
We would like to thank the reviewers of this paper, and the editor in charge (Elia Zardini), as well as Shawn Standefer for helpful comments and suggestions. Paul Egré thanks the ANR Program TrilLogMean ANR-14-CE30-0010-01 for funding, as well as Grants ANR-10-LABX-0087 IEC and ANR-10-IDEX-0001-02 PSL* for research carried out at the Department of Cognitive Studies of ENS. Robert van Rooij's thanks the ERC ESSENCE Project (FP7-PEOPLE-2013-ITN) and the NWO 'Language in Interaction' project for funding. Thanks also to the project Non-Transitive Logics: A New Approach to Paradoxes, funded by the MINECO (Government of Spain) Grant No.: FFI2013-46451-P.
}

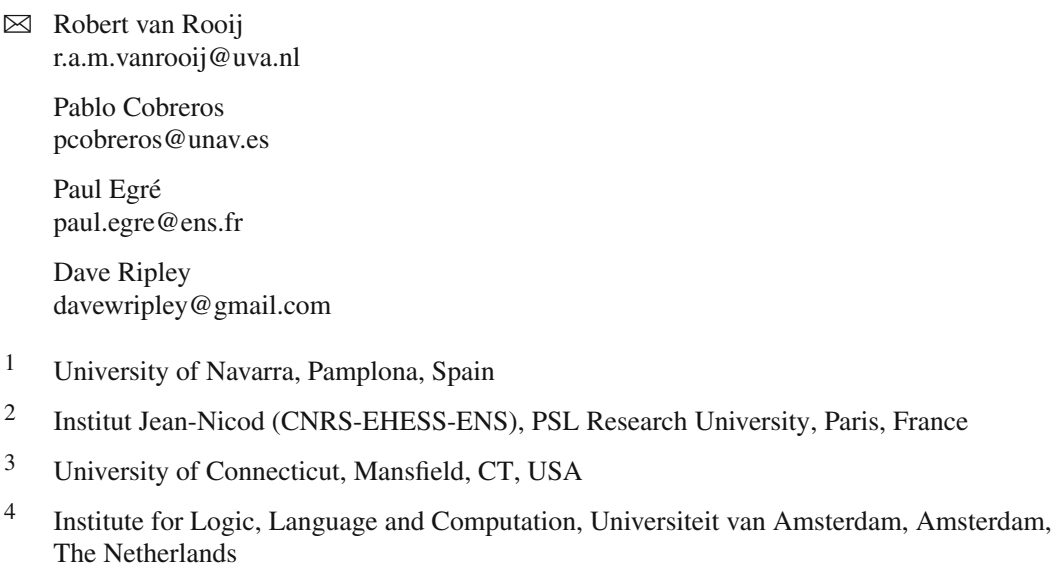


approaches and examine three specific consequence relations in relation to those, which we call: strict-to-tolerant entailment, pragmatic-to-tolerant entailment, and pragmatic-to-pragmatic entailment. The first two are nontransitive, whereas the latter two are nonmonotonic.

Keywords Sorites paradox · Vagueness · Strict-tolerant logic · Nontransitive consequence · Nonmonotonic consequence · Noncontractive consequence · Pragmatic reasoning

\section{Introduction}

According to one influential view of the sorites paradox, the tolerance principle - the constraint whereby if someone is tall, for example, then someone whose height is imperceptibly shorter is tall too-is an unsound rule of reasoning (see Williamson 1994). In combination with further premises that look less controversial, it leads to contradiction. We therefore ought to reject it, and not to rely on it when reasoning with vague predicates.

There are reasons to think of such a view as too drastic and as missing out on the role that such a principle plays in categorization and in ordinary judgmental and inferential practice (see van Rooij 2011b; Égré 2015). Taking a different perspective, the tolerance principle ought not to be discarded that fast, even when viewed normatively. Instead, it corresponds to what some authors have called a soft constraint (see Maudlin 2008), or a default (Cumming 2014), namely a rule that we can use legitimately in reasoning, but that cannot be used without some care.

One way to represent the tolerance principle is as a certain conditional sentence, of the form: $P a \wedge a \sim_{P} b \rightarrow P b$ (where $a \sim_{P} b$ means that $a$ and $b$ are similar in $P$-relevant respects, e.g. having similar heights relative to "tall"). Another way is as an argument, of the form: $P a, a \sim_{P} b \vdash P b$. Let us consider the conditional version first. Various options have been proposed to deal with the tolerance conditional as a soft constraint. In standard fuzzy logic, for example, the idea is to think of that conditional as not perfectly true, but close-to-perfectly-true. Let us assume the conditional to be Łukasiewicz's conditional (see Goguen 1969), and call a sentence close-to-perfectly-true provided its degree of truth is 0.9 . Given an appropriate sorites sequence (for example a series of individuals from clearly short to clearly tall, whose adjacent members differ imperceptibly in height), it will be possible to have: $P a_{1} \rightarrow P a_{2}$ close-to-perfectly-true, $P a_{2} \rightarrow P a_{3}$ close-to-perfectly-true, without having $P a_{1} \rightarrow P a_{3}$ close-to-perfectly-true. That is, each tolerance conditional is true to degree at least 0.9 , but the resulting conditional itself may end up with a degree of truth less than 0.9. If validity is defined as the preservation of perfect truth (degree 1) from premises to conclusions, modus ponens remains a valid rule, but the tolerance principle, though close-to-perfectly-true, is not true enough to produce a sound argument, in the sense of a valid argument based on perfectly true premises. Another possibility therefore is to define validity as the preservation of close-to-perfect-truth, for instance having a degree at least 0.9 . In that case the tolerance principle can be 
sound in the sense of being close-to-perfectly-true, but modus ponens is no longer valid, and the conditional is no longer transitive (Williamson 1994, p. 124). ${ }^{1}$

Further options for the definition of validity exist. Smith (2008) proposes that an argument is valid iff when all premises are strictly greater than 0.5 , the conclusion is greater or equal to 0.5 . The option is very similar to the one we pursue below. In particular it relies on a standard conditional connective, and yields ordinary classical logic. ${ }^{2}$

There is also the kind of definition of validity suggested in Edgington (1997). On this approach, an argument is valid iff the conclusion can never fall as far short of perfect truth as the sum of the extents to which the premises fall short of perfect truth. Edgington does not use Łukasiewicz's truth-degree conditions for her connectives, and so arrives at classical logic this way. But using Łukasiewicz's truth conditions, this would again result in a distinct logic, one that validates modus ponens but is noncontractive. We discuss noncontractive approaches to vagueness in Sect. 2.3.

An alternative to the fuzzy strategy is to view the tolerance conditional as expressing a defeasible rule. Say that $P a \wedge a \sim_{P} b \rightarrow P b$ is true provided $P b$ is true in all $\left(P a \wedge a \sim_{P} b\right.$ )-normal worlds [see Hu 2015; based on Asher and Morreau 1991]. Call a world $\left(\phi \wedge a \sim_{b} P\right.$ )-normal if $a$ is $P$-similar to $b$ but is not close to a borderline case of $P$. From $P a \wedge a \sim_{P} b, P a \wedge a \sim_{P} b \rightarrow P b$, it need not follow that $P b$, since a world may satisfy $P a \wedge a \sim_{P} b$ without being $\left(P a \wedge a \sim_{P} b\right)$-normal, precisely when $b$ is a borderline case of $P$. Again, this conditional fails to satisfy modus ponens, and it is also nontransitive. But moreover it is nonmonotone, since a world that is $P a \wedge a \sim_{P} b$-normal need not be $P a \wedge P c \wedge a \sim_{P} b$-normal. On that view, the tolerance conditional represents a defeasible rule, usable except in cases in which the main premise itself does not correspond to a normal world.

Both the fuzzy approach and the nonmonotonic approach have some appeal. On some understandings of fuzzy consequence, the sorites paradox is solved by saying that modus ponens is not valid. On the nonmonotonic approach, the sorites paradox is solved by saying that modus ponens is a defeasible rule: premises in sorites arguments are assertible, but the argument is not undefeasibly valid. However, the nonmonotonic approach faces a limitation, which concerns the treatment of the tolerance principle in terms of a special conditional connective. As is well known, a sorites argument can be stated using only conjunction and negation, by saying that it is not the case that there are two cases $a$ and $b$ that are very similar, but are such that $P a$ and not $P b$. But a nonmonotonic treatment of the conditional does not tell us how to address that alternative version of the sorites. The situation in fuzzy logic is better. Relative to Łukasiewicz's conditional, it is possible to define a strong conjunction \& such that the degree of truth of $(A \rightarrow B)$ is always equal to the degree of truth of $\neg(A \& \neg B) .^{3}$ However, the Łukasiewicz conditional loses some features that one might wish to

\footnotetext{
1 Williamson discusses a generalization of that notion of validity, as proposed by Machina (1976): an argument is valid iff the degree of the conclusion cannot be lower than the infimum of the degrees of the premises. This amounts to considering the preservation of close-to-prefect-truth over all possible thresholds; as such, modus ponens is not valid on this definition either.

2 We give a detailed comparison between our approach and Smith's approach in Cobreros et al. (2017).

${ }^{3}$ Łukasewicz's conditional is defined algebraically as $\min (1,1-x+y)$, and the strong conjunction or t-norm corresponding to it as $\max (x+y-1,0)$.
} 
retain for a conditional, such as contraction. Relative to the loss of modus ponens, this may not stand as a significant cost, but we would favor an approach that preserves more of the regular features of the standard conditional.

In this paper therefore, we are interested in accounts of vagueness that, instead of relying on a special conditional connective, propose to capture the tolerance principle by imposing specific constraints on the consequence relation, in a way that leaves intuitively desirable properties of a conditional connective in place, and in a way suitable to deal with the sorites argument in its conjunctive form as well as its conditional one. More specifically, we compare and discuss various structural choices that can be made on a consequence relation in order to make the tolerance principle valid, when represented not merely as a conditional sentence, but also as a rule, namely as an argument of form: $P a, a \sim_{P} b \vdash P b$. Our main focus is on two structural approaches which mirror the nontransitive and nonmonotone conditional to some extent, but shift those properties up one level, namely to the consequence relation. The first is the nontransitive treatment of logical consequence favored in our past work, on which the principle of tolerance comes out as valid in rule form, but cannot be iterated without risk. ${ }^{4}$ The second is the nonmonotone treatment of logical consequence, on which the principle of tolerance too can come out as valid, but in a way that is sensitive to context and to the addition of further premises (soft consequence as defeasible consequence) (see Misiuna 2010; Hu 2015).

This paper compares three notions of entailment, starting with strict-to-tolerant entailment (see Cobreros et al. 2012), which links the tolerance principle to nontransitive reasoning. We discuss two elaborations of $s t$-entailment, based on the idea of pragmatic strengthening. Those two variants differ in that they both treat the tolerance principle via nonmonotonic reasoning. The first of those variants combines nontransitivity and nonmonotonicity, whereas the second, though nonmonotonic, remains fully transitive. Our broader agenda is to compare those three notions, and to discuss their relative merits and limitations.

In Sect. 2, we start off with an overview of various structural choices that can be made on a consequence relation in order to block the sorites paradox, that is we survey several nontransitive, nonmonotonic, and noncontractive approaches. In Sect. 3, we focus on the nontransitive approach favored in our past work on strict-tolerant validity, and highlight some motivations for the definition of a generalized notion of pragmatic entailment on that basis, of which two variants are considered, which we call Prtentailment and $\operatorname{Pr} P r$-entailment. In Sect. 4, finally, we compare the three systems under discussion, and draw more general lessons for the modeling of reasoning with vague predicates.

\section{Transitivity, monotonicity, contraction}

In this section we consider three structural properties of particular interest in relation to soritical reasoning, namely transitivity, monotonicity, and contraction. Although noncontractive approaches will not play a role in our subsequent inquiry, they are

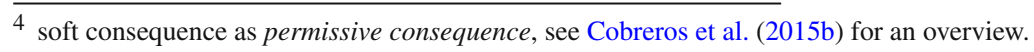


worth considering too, in particular because of some analogies with nontransitive treatments.

\subsection{Transitivity}

Here, we will call a consequence relation $\vdash$ 'transitive' iff whenever $\Gamma \vdash \phi, \Delta$ and $\Gamma^{\prime}, \phi \vdash \Delta^{\prime}$, then $\Gamma, \Gamma^{\prime} \vdash \Delta, \Delta^{\prime}{ }^{5}$ (We assume that $\Gamma$ and $\Gamma^{\prime}$ are read conjunctively, while $\Delta$ and $\Delta^{\prime}$ are read disjunctively.) Many consequence relations obey this condition (indeed, sometimes this is required as part of how 'consequence relation' is understood!); but in recent years, there has been increasing interest in systems that do not. (For examples, see Frankowski 2004; Hallnäs 1991; Hallnäs and SchroederHeister 1991; Tennant 1987; Weir 1998; Zardini 2008. Of these, only Zardini 2008 focus on vagueness.) We have ourselves explored several nontransitive systems in our past work, for example in Cobreros et al. (2012, 2013, 2015a); here, we focus on the kind of nontransitive approach to vagueness we recommend. ${ }^{6}$

On this approach, tolerance is not merely a formula that may occur or not in an argument; its effects are internalized in the consequence relation itself. In the system $s t$ of Cobreros et al. (2012), for example, $P a, a \sim_{P} b \vdash P b$. Note that no statement of tolerance occurs in this argument, and yet $s t$ validates it: the argument itself is a statement of tolerance. On this approach, the claim that $a$ is $P$, together with the claim that $a$ is $P$-similar to $b$, directly entails, without need for any further claims, the claim that $b$ is $P$. We are most interested in systems with this feature, whatever their structural properties, and we will aim to internalise tolerance into the consequence relations we explore throughout the paper.

If we combine this kind of principle with transitivity, we can reason as follows:

$$
\operatorname{Trans} \frac{P a_{0}, a_{0} \sim_{P} a_{1} \vdash P a_{1} \quad P a_{1}, a_{1} \sim_{P} a_{2} \vdash P a_{2}}{\operatorname{Trans} \frac{P a_{0}, a_{0} \sim_{P} a_{1}, a_{1} \sim_{P} a_{2} \vdash P a_{2}}{P a_{0}, a_{0} \sim_{P} a_{1}, a_{1} \sim_{P} a_{2}, a_{2} \sim_{P} a_{3} \vdash P a_{2}, a_{2} \sim_{P} a_{3} \vdash P a_{3}}}
$$

In this way, we can reach $P a_{0}, a_{0} \sim_{P} a_{1}, \ldots, a_{n-1} \sim_{P} a_{n} \vdash P a_{n}$ for any $n$. Call this ' $n$-fold tolerance'. $n$-fold tolerance seems to be a problem: the similarity premises here are exactly the setup for a sorites sequence for $P$, and this tells us that they entail, together with the claim that the first member of the sequence is $P$, that the last member is also $P$. That, of course, looks much like falling victim to the sorites paradox.

In a moment, we'll see that there are ways one might choose to live with $n$-fold tolerance, ways that do not fall victim to the paradox-but for now, let's assume it's

\footnotetext{
5 There is no single thing people mean when they call a consequence relation 'transitive', but this is one very standard understanding, and the differences don't matter for our purposes here. For some details, see Ripley (2017).

6 The approach of Zardini (2008) is similar to ours in many ways, and indeed forms part of the original inspiration for our approach. However, this approach does what we have said above we will not do: it introduces a new connective, not otherwise motivated, to play the role of the conditional in statements of tolerance. We set further comparison aside.
} 
to be avoided. The only ingredient here is transitivity itself; once tolerant reasoning is internalised in this way, there is no place else to push back on the above reasoning. One possible response, then, is to reject transitivity, to say that each individual step of soritical reasoning is indeed valid, but that chaining these valid steps together does not preserve their validity. ${ }^{7}$

This kind of approach allows appeal to tolerance, as internalised in the consequence relation, once but not more than once. This, then, is one way to understand tolerance's soft status; it can be appealed to freely, but we cannot combine the results of multiple appeals.

So much for internalised tolerance; what about formula-level statements of tolerance, like $\forall x \forall y((P x \wedge x \sim P \quad y) \rightarrow P y)$ ? If tolerance is really internalised in the consequence relation itself, you might expect such formulas to be theorems of the consequence relation: valid consequences of the empty set of premises. Indeed, this is exactly what happens in the systems we have explored elsewhere.

But you might also expect these tolerance formulas to be suppressible: to be such that, when an argument with a tolerance formula among its premises is valid, then the corresponding argument without that tolerance formula among its premises is also valid. ${ }^{8}$ When a consequence relation is transitive, in the sense we've given here, all of its theorems are suppressible, since the move from $\vdash A$ and $\Gamma, A \vdash \Delta$ to $\Gamma \vdash \Delta$ is just an instance of transitivity; but for nontransitive relations this need not be the case. (For the converse direction, it is reflexivity that matters; so long as each formula entails itself, suppressible formulas are theorems, since we can move from $A \vdash A$ to $\vdash A$ simply by suppressing $A$. We do not consider nonreflexive consequence relations here.)

In fact, tolerance formulas are not suppressible in the systems we have explored elsewhere; although tolerance is internalised in these consequence relations, still including a formula-level statement of tolerance as a premise can have an effect. This gives another way to understand tolerance's soft status. A consequence relation, at least when it is nontransitive, can give two kinds of endorsement to a formula: the weaker endorsement of theoremhood and the stronger one of suppressibility. Tolerance formulas receive the weaker endorsement but not the stronger; they are given, but not completely.

\subsection{Monotonicity}

A consequence relation $\vdash$ is monotonic iff whenever $\Gamma \vdash \Delta$, then $\Gamma, \Gamma^{\prime} \vdash \Delta, \Delta^{\prime}$; that is, if adding premises and/or conclusions to a valid argument can never result in an invalid argument. Nonmonotonic logics provide one of the most usual ways to understand defeasible reasoning, since they allow for validity to depend on the absence of certain information, and so to be defeated when such information arises. A standard example for this type of reasoning is the following: if a timetable does not mention

\footnotetext{
7 As we show in Cobreros et al. (2012), this is compatible with preserving the validity of every classicallyvalid argument; the breaks in transitivity can be restricted to those places where the tolerant aspect of the consequence relation has been appealed to repeatedly.

8 As far as we know, there is no standard term for what we are calling 'suppressible'; we take the term from (Routley et al. 1982, pp. 140ff.)'s discussion of 'suppression'.
} 
any direct flight from A to B, we routinely assume that no such flight exists, which, in turn, allows the planner to represent this information simply by not listing such a flight. This type of pragmatic reasoning is called in AI 'Reasoning as Failure' and is made use of in various nonmonotonic logics (e.g. McCarty's Circumscription and various variants of Logic Programming). It has been used as well in linguistics to account, among others, for the progressive (Asher 1992), generics (Asher and Morreau 1995), discourse structure (Asher and Lascarides 2003), several types of Gricean conversational implicatures (Schulz and van Rooij 2006), and even swear words (McCready 2012) (see Thomason 2011; van Rooij and Schulz 2011, for overview). The Sorites paradox is not standardly approached from a pragmatic point of view. It is perhaps not surprising, then, that there are not so many approaches to the sorites paradox based on nonmonotonic logics. However, there are some.

Approaches to tolerance that turn on rejecting monotonicity can be found in $\mathrm{Hu}$ (2015) and Misiuna (2010). ${ }^{9}$ Both Misiuna and Hu offer pictures involving new connectives to play the role of the conditional in tolerance principles, and so run afoul of the argument we've offered above involving conditional-free sorites. ${ }^{10}$ However, the way in which nonmonotonicity is involved can be maintained without a special conditional.

The basic idea behind these nonmonotonic approaches is to validate reasoning according to tolerance, but only when no countervailing information is available. That is, given only the premises $P a$ and $a \sim_{P} b$, we can validily conclude $P b$. Indeed, given the premises $P a_{0}, a_{0} \sim_{P} a_{1}, a_{1} \sim_{P} a_{2}, \ldots, a_{n-1} \sim_{P} a_{n}$, one may validly conclude $P a_{n}$; these approaches validate $n$-fold tolerance. ${ }^{11}$ However, in the presence of certain extra information-for example, the additional premises $a_{n} \sim_{P}$ $a_{n+1}$ and $\neg P a_{n+1}$ - this conclusion would not validly follow. As $\mathrm{Hu}$ (2015) puts the point, "sorites reasoning is...not...inherently defective or never worth utilizing, but...unproblematic in many instances, so long as it is not carried out too far down a sorites series".

This, then, gives a way to accept the validity of $n$-fold tolerance. The problem, on a nonmonotonic view, is not with concluding $P a_{n}$ on the basis of such a chain of tolerant reasoning; the problem occurs when such a conclusion is maintained in the face of countervailing evidence. Such countervailing evidence, of course, is present when we confront a sorites series; the puzzle is created precisely because we know that the last things in the series don't satisfy the relevant predicate. This is, then, the way in

\footnotetext{
9 The combination of nonmonotonicity with fuzzy logic has been explored in some artificial intelligence applications (for example in Ray and Chakraborty 2011; Zhang 2003), but these applications have not specifically addressed either tolerance or the sorites paradox, as far as we know.

Bennett (2006) includes a brief discussion of a nonmonotonic approach to the sorites, but without developing any details. Our account to follow fits nicely with the picture Bennett sketches, but other accounts easily could as well.

10 Indeed, $\mathrm{Hu}$ (2015) considers a version of this argument, and concedes in response that his account "fails to speak to the proper evaluation of the original sorites argument".

11 Misiuna (2010) claims that a similar argument is invalid in the system recommended there, but as far as we can see this is an error; the models $O$ that Misiuna calls 'most consistent' in $\$ 6$ do not actually seem to be.
} 
which we can understand tolerance's soft status within the nonmonotonic approach: tolerance can be appealed to repeatedly, but it gives way in the face of a sorites series.

How does this kind of nonmonotonic approach compare to the kind of nontransitive approach we have discussed above? Both agree that tolerance can be appealed to at the beginning of a sorites series, but not farther down. However, they see this in different ways. On a nontransitive approach, tolerance gives out from exhaustion as we proceed down such a series; it simply cannot go that far. On a nonmonotonic approach, it gives out only when it meets opposition; on its own it can proceed arbitrarily far.

There is another difference worth noting as well. Whereas the nontransitive approach allows for drawing conclusions that cannot be drawn on as premises, nonmonotonic approaches typically do not; for the nonmonotonician, once something cannot be drawn on as a premise it must also be withdrawn as a conclusion. ${ }^{12}$

\subsection{Contraction}

We will explore the third property-contraction-in less depth in the course of this paper, but we pause to present it here nonetheless, as it certainly provides another family of approaches worth considering. A consequence relation $\vdash$ is contractive iff: whenever $\Gamma, \phi, \phi \vdash \Delta$, then $\Gamma, \phi \vdash \Delta$, and whenever $\Gamma \vdash \phi, \phi, \Delta$, then $\Gamma \vdash \phi, \Delta$. (Good introductions to a variety of noncontractive logics can be found in Restall 2000; Paoli 2002; Bimbó 2015.)

In noncontractive logics, the number of times a premise or conclusion is appealed to may matter for validity. ${ }^{13}$ An argument that repeats a premise three times, say, may be valid, while a similar argument that only repeats the premise twice is not. This has been interpreted in a variety of ways; the way most relevant for our purposes is perhaps that of Slaney (2010). ${ }^{14}$

On the approach Slaney presents, the crucial failures of contraction involve precisely the tolerance principle. Note that, in a sorites argument with the tolerance principle as a premise, this principle must be appealed to many times-once for each step in the sorites series. This, then, provides us another way to think of tolerance as a soft or defeasible principle: perhaps it is a principle we may appeal to only a limited number

\footnotetext{
12 This is very like the difference (Hu 2015, fn. 37) sees between his account and the one of Kamp (1981).

13 The same is true of certain logics that fail monotonicity; where noncontractive logics can invalidate arguments with too few occurrences of some premise or conclusion, nonmonotonic logics can invalidate arguments with too many occurrences. Oversimplifying things, there are two nonmonotonic traditions: the default tradition and the substructural tradition; these traditions have some commonalities, but a large number of differences, not least in intended applications. The former tradition, which is what we have focussed on in Sect. 2.2, tends not to care about number of occurrences in this way; in default logics, adding new formulas to a valid argument can result in a invalid argument, but adding new occurrences of formulas that are already present cannot. However, the latter tradition pays a great deal of attention to number of occurrences. For an overview of this aspect of relevant logics, see Meyer and McRobbie (1982a, b); in other nonmonotonic logics of the substructural tradition the situation is similar.

14 Note that a variety of many-valued approaches to vagueness, based on Łukasiewicz and related logics, are in fact noncontractive if looked at in a certain light. These include the approaches of Lakoff (1973), Machina (1976) and Slaney (2010). For useful discussion, see Paoli (2003). Recall the Introduction.
} 
of times in any argument, a principle that holds once (or twice, or three times) but not an unlimited number of times.

Of course, to fully make sense of this we would need to have an understanding of what it is for something to hold only once (or twice, or three times). We will not attempt to make this idea precise here. The rough idea, however, is that in the absence of contraction, we can allow for principles like tolerance, which are meant to be defeasible, to be appealed to a limited number of times. When they are defeated, in a setting like this one, it is in a battle of attrition.

These noncontractive approaches share a certain flavour with nontransitive approaches: according to both, we have something that can be appealed to without any worry or caveat, but only a limited number of times. On nontransitive approaches, it is the consequence relation itself that is like this; on noncontractive approaches, it is the tolerance principle.

Uniquely among the three kinds of structural approach we consider, there does not seem to be a way for a noncontractive approach to internalise tolerance in the consequence relation itself, at least not if the sorites is to be addressed by failures of contraction. The reason is that noncontractive consequence relations can only prevent multiple uses of things that actually appear as premises or conclusions, but tolerance itself is the only thing appealed to repeatedly in a sorites argument. If this repeated appeal is to be blocked, then, tolerance cannot be internalised; it must appear explicitly. For this reason, we set noncontractive approaches to tolerance aside for the remainder of the paper.

\section{From nontransitive to nonmonotonic}

In previous work we showed how can we endorse tolerance as a soft constraint within the context of non-transitive entailment. This way of endorsing tolerance has the consequence, to which we pointed out above, of making a difference between the endorsement of a formula as a theorem and as a suppressible premise. In our nontransitive framework tolerance, as explicit formula, is endorsed in the former but not in the latter sense. But there are reasons for which one would like to endorse tolerance in a stronger sense; for example, to be able to properly assert tolerance in some contexts. In a relatively independent discussion, in response to objections raised in Alxatib et al. (2013), we developed a notion of pragmatic interpretation. In this section we show how this can be used to go from our original non-transitive account of tolerance to a non-monotonic endorsement of tolerance.

\subsection{The logic $s t$}

Let $\mathcal{M}=\langle\mathcal{D}, \mathcal{I}\rangle$, with $\mathcal{I}$ a total function from atomic sentences to $\left\{0, \frac{1}{2}, 1\right\}$. This model extends to formulas according to the strong Kleene valuation scheme: ${ }^{15}$

\footnotetext{
15 We assume here for convenience that each $d \in D$ has a name $\underline{d}$; this bears no weight in the account.
} 
- $\mathcal{V}_{\mathcal{M}}(\phi)=\mathcal{I}_{\mathcal{M}}(\phi)$, if $\phi$ is atomic.

- $\mathcal{V}_{\mathcal{M}}(\neg \phi)=1-\mathcal{V}_{\mathcal{M}}(\phi)$.

- $\mathcal{V}_{\mathcal{M}}(\phi \wedge \psi)=\min \left\{\mathcal{V}_{\mathcal{M}}(\phi), \mathcal{V}_{\mathcal{M}}(\psi)\right\}$.

- $\mathcal{V}_{\mathcal{M}}(\phi \vee \psi)=\max \left\{\mathcal{V}_{\mathcal{M}}(\phi), \mathcal{V}_{\mathcal{M}}(\psi)\right\}$.

- $\mathcal{V}_{\mathcal{M}}(\forall x \phi)=\min \left\{\mathcal{V}_{\mathcal{M}}\left(\left[\frac{d}{d} / x\right] \phi\right): d \in D\right\}$.

We say that $\phi$ is strictly true in $\mathcal{M}$ iff $\mathcal{V}_{\mathcal{M}}(\phi)=1$, and that $\phi$ is tolerantly true in $\mathcal{M}$ iff $\mathcal{V}_{\mathcal{M}}(\phi) \geq \frac{1}{2}$. In terms of this semantics we can define some well-known logics: Kleene's $K 3$ and Priest's $L P$. Both logics understand entailment as preservation of truth in all models, the difference is that while for $K 3$ truth it means strict truth, for $L P$ it means tolerant truth:

$$
\begin{gathered}
\Gamma \vDash_{K 3} \Delta \text { just in case for all } \mathcal{M}: \\
\text { if } \forall \gamma \in \Gamma: \mathcal{V}_{\mathcal{M}}(\gamma)=1 \text {, then } \exists \delta \in \Delta: \mathcal{V}_{\mathcal{M}}(\delta)=1 . \\
\Gamma \models_{L P} \Delta \text { just in case for all } \mathcal{M}: \\
\text { if } \forall \gamma \in \Gamma: \mathcal{V}_{\mathcal{M}}(\gamma)>0, \text { then } \exists \delta \in \Delta: \mathcal{V}_{\mathcal{M}}(\delta)>0 .
\end{gathered}
$$

A fundamental idea in Cobreros et al. (2012) to define entailment from strict to tolerant:

$$
\begin{gathered}
\Gamma \models^{s t} \Delta \text { just in case for all } \mathcal{M}: \\
\text { if } \forall \gamma \in \Gamma: \mathcal{V}_{\mathcal{M}}(\gamma)=1 \text {, then } \exists \delta \in \Delta: \mathcal{V}_{\mathcal{M}}(\delta)>0 .
\end{gathered}
$$

Thus, although we don't give up the idea that entailment is truth-preserving, we allow the standard of assertion of the conclusions to be weaker than the standard of assertion of the premises. A surprising feature of this logic is that although the semantics makes use of three truth-values, the consequence relation is exactly the familiar consequence relation of classical logic. This fact contrasts sharply with $K 3$ and $L P$, both of which give up a good amount of classically valid arguments.

Now, despite its classicality, this new semantics makes room for tolerance without falling victim to the sorites paradox. In order to account for tolerance, we extend the language with similarity relations, $\sim_{P}$, one for each predicate $P$. There are various ways to interpret this relation that would work for our purposes. One interpretation is the following, taken up in Cobreros et al. (2015a):

- $\mathcal{V}_{\mathcal{M}}\left(a \sim_{P} b\right)=1$ iff $\left|\mathcal{V}_{\mathcal{M}}(P a)-\mathcal{V}_{\mathcal{M}}(P b)\right|<1,0$ otherwise.

The resulting logic $s t^{\sim}$ is a conservative extension of classical logic, in the sense that any classically valid argument in the old vocabulary remains valid. In addition, the tolerance formula $\left(\forall x, y\left(\left(P x \wedge x \sim_{P} y\right) \rightarrow P y\right)\right)$ becomes valid, as does the tolerance argument: $P a, a \sim_{P} b \vDash_{\sim}^{s t} P b .{ }^{16}$ The endorsement of tolerance does not lead to paradox, however, since tolerance in $s t^{\sim}$ leads to non-transitivity:

\footnotetext{
16 Ripley (2013) and Cobreros et al. (2013) show a similar result for a language with a transparent truthpredicate.
} 


$$
\begin{gathered}
P a, a \sim_{P} b \models_{\sim}^{s t} P b \text { and } P b, b \sim_{P} c \models_{\sim}^{s t} P c \\
\text { BUT } \\
P a, a \sim_{P} b, b \sim_{P} c \not \nvdash_{\sim}^{s t} P c .
\end{gathered}
$$

We felt, and still feel, that this is a very intuitive and appealing treatment of the sorites paradox. The treatment however, comes with the limitation that we should make a distinction about the diagnosis of the sorites paradox depending on its formulation. If we look at the sorites as a step-by-step argument based on the validity of tolerance inferences then, though each tolerance inference is valid, validity breaks when we try to chain these inferences. If we consider the sorites argument with the tolerance formula $\left(\forall x, y\left(\left(P x \wedge x \sim_{P} y\right) \rightarrow P y\right)\right)$ as an explicit premise, then although that formula is valid, the resulting argument is valid but unsound; it fails to establish its conclusion, since tolerance cannot be freely drawn on as a premise. In short, although the tolerance formula is valid, according to the logic $s t^{\sim}$ we are not in a position to draw on it as a premise without further ado. However, there are contexts in which we would like to assert the tolerance formula and go on to draw conclusions from it. That is, we would like to assert it strictly. But there are other assertions we might want to make that signal only that something can be validly concluded, not that it may be drawn on as a premise. In these cases, we want to assert tolerantly. That is, when we make an assertion we would like to interpret it sometimes tolerantly and sometimes strictly.

A number of recent experiments (e.g., Alxatib and Pelletier 2011; Ripley 2011; Égré et al. 2013; Egré and Zehr 2016) show that naive speakers find a logical contradiction like 'John is tall and John is not tall' acceptable in cases where John is a borderline tall man. This seems to show that we need to take account of tolerant truth, since tolerant truth exhibits this exact behaviour. However, just relying on the notion of tolerant truth would mean that the assertion 'John is tall' would be acceptable in the same situation. The same experimental evidence shows, however, that this is not the case: 'John is tall' is taken to be acceptable only if John is really tall. In terms of our three-valued models this could be modeled by saying that the assertion 'John is tall' is acceptable only if John is strictly tall. Similarly, Serchuk et al. (2011) found that classical tautologies like ' $T j \vee \neg T j$ ' are generally not accepted if John is borderline tall. So making use not only of tolerant, but also of strict truth (which exhibits this latter behavior) seems required.

The conclusion we draw from the previous discussion, together with considerations raised in Cobreros et al. (2012, 2015a), is that we should interpret a sentence strictly if possible, and tolerantly otherwise. This interpretation strategy is in line with a usual strategy to account for scalar implicatures: where Grice (1967) assumed that from an assertion of the form ' $\phi \vee \psi$ ' one can conclude that it is not the case that $\phi \wedge \psi$ is true, because otherwise the speaker would have said so, we can assume that from assertion ' $\phi$ ' one can conclude that $\phi \wedge \neg \phi$ is not true for a similar reason. The strategy to interpret strictly if possible, and tolerantly otherwise, has just this effect.

Unfortunately, this interpretation strategy taken at face value gives rise to trouble for more complex sentences. Alxatib et al. (2013) show that this wrongly predicts that a sentence like 'Adam is tall and not tall, or John is rich' entails that John is strictly rich, although it should not entail this and intuitively should mean that either Adam 
is borderline tall or John is strictly rich. In Cobreros et al. (2015b) we responded by providing a more sophisticated pragmatic interpretation rule to strengthen the meaning of a sentence, which we review below. ${ }^{17}$

\subsection{Pragmatic interpretation}

\subsubsection{Truth-makers}

To account for this pragmatic strengthening we will make use of truth-makers. We propose that the pragmatic interpretation of $\phi$ makes one exact truth-maker of $\phi$ as true as possible. To determine what the truth-makers of a sentence are, we follow van Fraassen (1969).

We think of a state of affairs as a situation that might hold or might not hold. The state of a affairs that Socrates is wise holds iff the sentence 'Socrates is wise' is true. The state of a affairs Socrates is not Cretan holds if the sentence 'Socrates is not Cretan' is true. ${ }^{18,19}$ A fact is any non-empty set of states of affairs. We call facts that contains only a single state of affairs, like \{Socrates is wise\}, an atomic fact. This atomic fact is a truth-maker for the sentence 'Socrates is wise' but also the conjunctive fact \{Socrates is wise, Socrates is not Cretan\} is a truth-maker for that sentence.

More generally, let $S O A$ be the set of all states of affairs. For each state of affairs $\mathbf{p} \in S O A$ there is a corresponding complement $\overline{\mathbf{p}} \in S O A$ for which it holds that $\overline{\overline{\mathbf{p}}}=\mathbf{p}$. We assume for simplicity a close correspondence between atomic sentences of the language and states of affairs: there is exactly one state of affairs corresponding to each literal. The set of facts, $\mathcal{F}$, is $\wp(S O A)-\emptyset$ : that is, a fact is a non-empty set of states of affairs. If $\mathbf{p}, \mathbf{q} \in S O A$, then $\{\mathbf{p}\}$ and $\{\mathbf{q}\}$ are atomic facts, and $\{\mathbf{p}, \mathbf{q}\}$ is a conjunctive fact. A truth-maker for a sentence $\phi$ (or the proposition expressed by that sentence) is a fact that makes $\phi$ true. We follow van Fraassen further in saying that an atomic sentence might have more than one truth-maker. An atomic sentence $p$ is not only made true by atomic fact $\{\mathbf{p}\}$, but also by conjunctive fact $\{\mathbf{p}, \mathbf{q}\}$. The former is a more minimal truth-maker than the latter. More interestingly, disjunctive sentences might have several minimal truth-makers. The disjunction $p \vee q$, for instance, has two minimal truth-makers: $\{\mathbf{p}\}$ and $\{\mathbf{q}\}$. What we are after, however, is the notion of what Fine (2014) calls the exact truth-makers for $\phi$. We say that the disjunction $p \vee(p \wedge q)$-although it has only $\{\mathbf{p}\}$ as its minimal truth-maker-has two exact truth-makers: $\{\mathbf{p}\}$ and $\{\mathbf{p}, \mathbf{q}\}$. Following van Fraassen (1969), we give the following

\footnotetext{
17 Other pragmatic strategies can be proposed that would agree with the idea of interpreting a sentence strictly if possible. See Egré and Zehr (2016) for a proposal to compute pragmatic strengthening more locally, and for comparisons with the algorithm in Cobreros et al. (2015b). Both approaches can answer the challenge raised by Alxatib et al, but they make different predictions in other specific cases.

18 If one doesn't like states of affairs, one can always think of them in a purely linguistic way simply as literals: atomic sentences and their negations.

19 One might think that we don't really have negative state of affairs, that $\neg p$ is true not because $\overline{\mathbf{p}}$ is the case, but rather because some $\mathbf{q}$ is the case that is incompatible with $\mathbf{p}$. On such an approach, 'This is green' might have more than one falsity-maker: 'This is red', 'This is blue', etc. For related ideas, see Millikan (1984, Ch. 14). Although such an analysis of falsity would be interesting, for simplicity we stick with van Fraassen's original proposal.
} 
simultaneous recursive definition of the set of exact truth-makers of $\phi, T(\phi)$, and the set of exact falsity-makers, $F(\phi):{ }^{20}$

\begin{tabular}{ll}
\hline$T(p)=\{\{\mathbf{p}\}\}$ & $F(p)=\{\{\overline{\mathbf{p}}\}\}$ for atomic $p$ \\
$T(\neg \phi)=F(\phi)$ & $F(\neg \phi)=T(\phi)$ \\
$T(\phi \wedge \psi)=T(\phi) \otimes T(\psi)$ & $F(\phi \wedge \psi)=F(\phi) \cup F(\psi)$ \\
$=\{X \cup Y \mid X \in T(\phi), Y \in T(\psi)\}$ & \\
$T(\phi \vee \psi)=T(\phi) \cup T(\psi)$ & $F(\phi \vee \psi)=F(\phi) \otimes F(\psi)$ \\
\hline
\end{tabular}

Notice that according to these rules, $T(p)=\{\{\mathbf{p}\}\}, T(\neg p)=\{\{\overline{\mathbf{p}}\}\}, T(p \vee q)=$ $\{\{\mathbf{p}\},\{\mathbf{q}\}\}$ and $T(p \wedge q)=\{\{\mathbf{p}, \mathbf{q}\}\}$. We analyse conditionals like $\phi \rightarrow \psi$ as material implication, that is $p \rightarrow q \equiv \neg p \vee q$ and $\neg(p \wedge q) \equiv \neg p \vee \neg q$, and thus $T(p \rightarrow$ $q)=\{\{\overline{\mathbf{p}}\},\{\mathbf{q}\}\}$ and $T(\neg(p \wedge q))=\{\{\overline{\mathbf{p}}\},\{\overline{\mathbf{q}}\}\}$.

To account for quantifiers, we first have to spell out how we interpret atomic sentences with predicates and individual terms. To do so, we assume that for each $n$-place predicate $P$ the model contains states of affairs like $\mathbf{P} \mathbf{d}_{\mathbf{1}}, \ldots \mathbf{d}_{\mathbf{n}}$, with each $\mathbf{d}_{\mathbf{i}} \in D$ an individual. We assume for simplicity that each $\mathbf{d} \in D$ has a unique name $\underline{d}$ in the language.

- $T\left(P \underline{d_{1}}, \ldots, \underline{d_{n}}\right)=\left\{\left\{\mathbf{P d}_{\mathbf{1}}, \ldots, \mathbf{d}_{\mathbf{n}}\right\}\right\}$

- $T(\forall \overline{x \phi})=-\bigotimes_{d \in D} T\left(\phi\left[^{x} / \underline{d}\right]\right)$

- $T(\exists x \phi)=\bigcup_{d \in D} T\left(\phi\left[^{x} / \underline{d}\right]\right)$

$$
\begin{aligned}
& F\left(P d_{1}, \ldots, d_{n}\right)=\left\{\left\{\overline{\mathbf{P d}_{\mathbf{1}}, \ldots, \mathbf{d}_{\mathbf{n}}}\right\}\right\} \\
& F(\forall x \phi)=\overline{\bigcup_{d \in D} F\left(\phi\left[^{x} / d\right]\right)} \\
& F(\exists x \phi)=\bigotimes_{d \in D} F\left(\phi\left[{ }^{x} / \underline{d}\right]\right)
\end{aligned}
$$

Observe that $T(\forall x P x)=T(P \underline{a}) \otimes T(P \underline{b})=\{\{\mathbf{P a}, \mathbf{P b}\}\}$, if $D=\{a, b\}$. Similarly, $T(\exists x P x)=T(P \underline{a}) \cup T(P \underline{b})=\{\{\mathbf{P a}\},\{\mathbf{P b}\}\}$. Notice that facts might not only be incomplete (neither verify nor falsify a sentence), they might also be inconsistent and both verify and falsify a sentence. Indeed, we have not ruled out facts like $\{\mathbf{T j}, \overline{\mathbf{T j}}\}$. Such inconsistent facts are crucial for us to model the meaning of vague sentences, expressing in this case that John is borderline tall.

$T(\phi)$ can be thought of as a fine-grained semantic interpretation of $\phi$. It can be used to determine a truth-conditional meaning for $\phi$ in terms of possible worlds, so long as we have some set $W$ of worlds, understood as special facts:

$$
\llbracket \phi \rrbracket \stackrel{d f}{=}\{w \in W \mid \exists f \in T(\phi): f \subseteq w\} .
$$

It is usual, however, to suppose that not just any fact counts as a world; and depending on what restrictions are or are not imposed, this truth-conditional meaning can behave in different ways. ${ }^{21}$ On one standard approach to worlds that fits with this setup, worlds are taken to be complete and consistent facts. That is, a world is a set $w$ of states of

\footnotetext{
${ }^{20}$ Note that the definition of $T(\phi)$ parallels the construction of the disjunctive normal form of $\phi$ : truthmakers basically correspond to disjuncts of such a normal form, and their members to conjuncts of the corresponding conjunctions.

${ }^{21}$ One might consider the totally unrestricted case, modeling the meaning of a sentence as the set of all facts that make it (perhaps inexactly) true. If $F$ is the set of all facts, we can define the following semantic Footnote 19 continued
} 
affairs such that for each atomic sentence $p$ in the language, either $\mathbf{p} \in w$ or $\overline{\mathbf{p}} \in w$ and not both. For our purposes, however, this will not work; it gives us no way to capture the difference between strict and tolerant satisfaction at a world, and so prevents us from connecting this approach to our initial three-valued setup. We will instead take worlds to be complete facts, with no requirement of consistency. That is, a world is a set $w$ of states of affairs such that for each atomic sentence $p$ in the language, either $\mathbf{p} \in w$ or $\overline{\mathbf{p}} \in w$ (and possibly both). This allows us to capture the difference between strict and tolerant satisfaction at a world, connecting truth-maker semantics to our three-valued $s t$-models above. For each atomic sentence $p$ and world $w$, we define:

$$
\begin{array}{ll}
\mathcal{V}_{w}(p)=1 & \text { iff } \quad \mathbf{p} \in w \text { and } \overline{\mathbf{p}} \notin w . \\
\mathcal{V}_{w}(p)=0 & \text { iff } \quad \mathbf{p} \notin w \text { and } \overline{\mathbf{p}} \in w . \\
\mathcal{V}_{w}(p)=\frac{1}{2} & \text { iff } \quad \mathbf{p} \in w \text { and } \overline{\mathbf{p}} \in w .
\end{array}
$$

These values can then be used just as outlined above to define the tolerant and the strict semantic interpretation of any sentence.

\subsubsection{Pragmatic meaning}

However, we did not introduce truth-makers just to recover notions we already had. Our purpose in introducing truth-makers is to define a notion of pragmatic meaning in terms of which we can strengthen the semantic meaning of a sentence. ${ }^{22}$ We have suggested at the end of the previous section that although we allow for inconsistencies, we can still pragmatically infer that $\neg p$ is not true from the fact that ' $p$ ' is asserted, by a reasoning analogue to the kind of reasoning involving scalar implicatures. In linguistic pragmatics it is not uncommon to use minimal models (we will not bother to distinguish models from worlds, using the terms interchangeably) to account for scalar pragmatic implicatures (e.g. Schulz and van Rooij 2006). We will do the same here to strengthen the semantic meaning, although we will think of a minimal world, in a somewhat different way. For us, a minimal world that makes $\phi$ true is one that is minimally inconsistent: it doesn't contain more inconsistencies than required to make $\phi$ at least tolerantly true. It's worth considering a few ways we might try to get to grips with this intuitive notion. The first way to model this that comes to mind is the following definition, with $v<w \operatorname{iff}_{d f}\{x \in S O A:\{x, \bar{x}\} \subseteq v\} \subsetneq\{x \in S O A:\{x, \bar{x}\} \subseteq w\}$ :

- $\operatorname{Prag}(\phi)=\{w \in \llbracket \phi \rrbracket \mid \neg \exists v \in \llbracket \phi \rrbracket: v<w\}$.

notion of meaning: $[[\phi]] \stackrel{d f}{=}\{g \in F \mid \exists f \in T(\phi): f \subseteq g\}$. It is in terms of this notion of meaning that van Fraassen (1969) provides a semantics for the notion of tautological entailment: $\phi \vDash^{t e} \psi$ iff $_{d f}$ $[[\phi]] \subseteq[[\psi]]$. Notice that $[[\phi]]]$ might be thought of as the set of (possibly non-total) situations at which $\phi$ is true. It is $[[\phi]]$ rather than $T(\phi)$ that Kratzer and others take to be the fine-grained semantic meaning of a sentence in Situation Semantics (see Kratzer 2016). Although $T(\phi) \subseteq[[\phi]]$, and all elements of $[[\phi]]]$ make $\phi$ true, only those in $T(\phi)$ make $\phi$ exactly true.

${ }^{22}$ For a closely related analysis of 'scalar' implicatures using facts, see van Rooij (2017). 
Indeed, this notion of minimally inconsistent interpretation is used in Priest (1991). Unfortunately, as discussed in Cobreros et al. (2015b), this interpretation rule won't do for our purposes. Recall that according to Cobreros et al. (2012), to communicate that John is a borderline case of a tall man one can say 'John is tall and not tall'. According to the above pragmatic interpretation, however, a sentence like 'John is tall and not tall, or Mary is rich' is incorrectly interpreted as being pragmatically equivalent with 'Mary is rich'; the contradictory disjunct is too hastily ruled out (see Alxatib et al. 2013).

To avoid this prediction, we argued in Cobreros et al. (2015b) for the following definition of pragmatic interpretation (where $v<_{f} w \quad$ iff $_{d f} \quad\{x \in S O A: x \in$ $f \& \bar{x} \in v\} \subsetneq\{x \in S O A: x \in f \& \bar{x} \in w\})$ :

$$
P R A G(\phi)={ }_{d f} \quad\left\{w \in W \mid \exists f \in T(\phi): f \subseteq w \& \neg \exists v \supseteq f: v<_{f} w\right\} .
$$

The function $P R A G$ differs in two important ways from Prag: (1) PRAG( $\phi)$ looks for minimal worlds for each exact truth-maker of $\phi$ rather than for the whole sentence itself, and (2) for each of these exact truth-makers, it only seeks to minimize inconsistencies with respects to the atomic facts occurring in it; it doesn't bother about other inconsistencies. It is due to the first feature that we achieve the correct prediction for "John is tall and not tall, or Mary is rich"; the exact truth-maker for this sentence that makes its first disjunct true is inconsistent, and so no world containing it could occur in the sentence's Prag-value, since there is another exact truth-maker that is consistent. But $P R A G$ allows such inconsistent worlds to occur.

$P R A G$ gets many other predictions correct as well: (1) 'John is tall' is pragmatically interpreted to mean that John is strictly tall (that is, $w \in P R A G(T j)$ iff $\mathcal{V}_{w}(T j)=1$ ), (2) 'John is not tall' is predicted to mean that John is not even tolerantly tall ( $w \in$ PRAG $(\neg T j)$ iff $\mathcal{V}_{w}(T j)=0$ ), (3) 'John is tall and John is not tall' means that John is borderline tall, i.e. tolerantly tall but not strictly tall ( $w \in P R A G(T j \wedge \neg T j)$ iff $\mathcal{V}_{w}(T j)=\frac{1}{2}$ ), and (4) 'John is tall or not tall' means that John is not borderline tall ( $w \in$ $P R A G(T j \vee \neg T j)$ iff $\mathcal{V}_{w}(T j)=1$ or 0$)$. Those predictions are in accordance with recent experimental results reported by Alxatib and Pelletier (2011), Ripley (2011), Serchuk et al. (2011), Égré et al. (2013) and Egré and Zehr (2016). Furthermore, 'John is tall and not tall, and Mary is rich' is pragmatically interpreted to mean that John is borderline tall and Mary strictly rich, which seems intuitively correct. Finally, 'John is tall and not tall, or Mary is rich' is correctly interpreted as saying that John is borderline tall, or Mary is strictly rich.

\subsubsection{A tolerance constraint}

Let us go back now to the tolerance principle. Since similarity statements like $a \sim_{P} b$ are atomic, they correspond to atomic SOAs like $\mathbf{a} \sim_{\mathbf{P}} \mathbf{b}$. At the moment, however, our worlds pay no attention to the connection between these SOAs and the SOAs they are meant to constrain, like $\mathbf{P a}$ and $\mathbf{P b}{ }^{23}$ We need to implement some kind of

\footnotetext{
23 There are lots of different reasons one might believe in such a constraint, and those might affect more detailed models at exactly this point. For example, whether John is tall is presumably a matter (at least in part) of his height; but so too is whether he is 'tall'-similar to Mary. This might push for involving heights
} 
constraint so that tolerance can be respected. Here is our chosen constraint: we require that $\mathcal{V}_{w}\left(a \sim_{P} b\right)=1-\left|\mathcal{V}_{w}(P a)-\mathcal{V}_{w}(P b)\right|$. That is, $a$ and $b$ are to count as $P$-similar to the extent that the value of $P a$ matches the value of $P b \cdot{ }^{24}$ We henceforth exclude all worlds $w$ that violate this condition.

One immediate effect of this is that the tolerance conditionals $P a \wedge a \sim_{P} b \rightarrow P b$ come out at least tolerantly true at every world. For a world to assign such a conditional the value 0 , it would need to assign both $P a$ and $a \sim_{P} b$ the value 1 and $P b$ the value 0 . But this is not possible compatibly with the constraint; if a world assigns 1 to $a \sim_{P} b$, then it must assign the same value to $P a$ and $P b$. These tolerance conditionals, then, can never receive the value 0 at any world.

\subsection{Tolerance and inference relations}

\subsubsection{Pragmatic-to-tolerant entailment ( Prt)}

Our pragmatic interpretation rule can be included in the definition of logical consequence to try and overcome the limitations we pointed out above about the assertability of the tolerance formula in $s t^{\sim 25}$ In Cobreros et al. (2015b), we introduced the following notion of pragmatic consequence, $\models^{p r t}$, going from pragmatically strongest to tolerant:

$$
\text { - } \Gamma \vDash \models^{p r t} \phi \quad \operatorname{iff}_{d f} \bigcap_{\psi \in \Gamma} P R A G(\psi) \subseteq \llbracket \phi \rrbracket
$$

The resulting notion of consequence, however, distinguishes between separate premises and their conjunction. There are cases in which $\phi, \psi \vDash^{p r t} \chi$ but $\phi \wedge \psi \not \nvdash^{p r t}$ $\chi$. For example, $p, \neg p \models^{p r t} q$ : worlds in $P R A G(p)$ must make $p$ strictly true, and worlds in $P R A G(\neg p)$ must make it strictly false, so $P R A G(p) \cap P R A G(\neg p)=\emptyset$. However, $p \wedge \neg p \not \models p r t q$, as $P R A G(p \wedge \neg p)$ is not empty, and includes some worlds outside of $\llbracket q \rrbracket$. The trouble is that $P R A G$ here has its effect only on individual sentences, when we might want it to apply to full collections of premises. To that end, we introduce the following variant approach. We restrict attention to finite sets of premises, and say that a finite set of premises $\Gamma$ entails $\phi$ provided the pragmatic meaning of the conjunction of the premises entails the tolerant meaning of the conclusion. (For empty

\section{Footnote 21 continued}

in our SOAs. Or, it might be that whether John is 'tall'-similar to Mary is a matter (at least in part) of the range of contexts in which both John and Mary count as tall, and in which neither John nor Mary count as tall. This might push for involving contexts in our SOAs. See Wright (1975), Kennedy and McNally (2005), van Rooij (2011b) and Burnett (2014) for discussions of related ideas. Here, though, we take no stand on these issues; we implement the connection between $\sim_{P}$ and $P$ directly, rather than offering any theory of why the connection holds.

24 This has some perhaps-counterintuitive results. For example, it makes everything that is $P$ to value 1 $P$-similar; but we might think that someone 2 meters tall is not 'tall'-similar to someone 3 meters tall, even though both are certainly tall. But let this pass; the constraint is simple and easy to work with; if it needs to be changed eventually, it is still a workable starting point.

25 This is similar to a standard approach in nonmonotonic logics. In McCarthy (1980)'s circumscription theory, for instance, $\phi \mid \sim \psi$ iff all models making the circumscribed interpretation of $\phi$ true also make $\psi$ true. For a use of this kind of consequence relation to account for pragmatic reasoning, see Schulz and van Rooij (2006). 


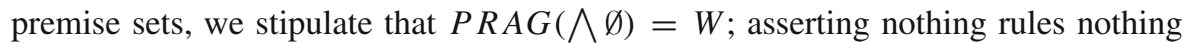
out.) We call the corresponding notion of validity Prt, and in what follows, we focus on Prt rather than prt, although there is much that is similar about them:

\section{- $\Gamma \vDash^{P r t} \phi \quad \operatorname{iff}_{d f} \quad P R A G(\bigwedge \Gamma) \subseteq \llbracket \phi \rrbracket$}

We want to call quick attention to three features of Prt. First, Prt has all tolerance formulas as theorems, since for each such formula $A$, $\llbracket A \rrbracket=W$, as we saw in Sect. 3.2.3. Second, $\phi, \neg \phi \not \nvdash^{P r t} \psi$; since there can be worlds that tolerantly satisfy $\phi \wedge \neg \phi$, and such worlds need not satify all $\psi$ even tolerantly, explosion is not valid and Prt entailment is paraconsistent. Third, conjunction elimination is valid: $\phi \wedge \psi \vDash{ }^{p r t} \phi$ and $\phi \wedge \psi \vDash p r t \psi$, since a world must at least tolerantly satisfy something in order to pragmatically satisfy it, and tolerant satisfaction of a conjunction requires tolerant satisfaction of each conjunct.

We turn now to Prt's structural properties. The first thing to note is that Prt is nonmonotonic, and in ways we might want for handling soritical situations. To start, Prt validates $n$-fold tolerance: $P a_{0}, a_{0} \sim_{P} a_{1}, \ldots, a_{n-1} \sim_{P} a_{n} \vDash^{P r t} P a_{n}$. To see this, consider a world $w \in P R A G\left(P a_{0} \wedge a_{0} \sim_{P} a_{1} \wedge \cdots \wedge a_{n-1} \sim_{P} a_{n}\right)$. There is only one fact in $T\left(P a_{0} \wedge a_{0} \sim_{P} a_{1} \wedge \cdots \wedge a_{n-1} \sim_{P} a_{n}\right)$, and it is consistent; thus, any such $w$ must give $\mathcal{V}_{w}\left(P a_{0}\right)=\mathcal{V}_{w}\left(a_{0} \sim_{P} a_{1}\right)=\cdots=\mathcal{V}_{w}\left(a_{n-1} \sim_{P} a_{n}\right)=1$. But since all the similarity facts take value 1 , by our constraint on worlds, we must have $1=\mathcal{V}_{w}\left(P a_{0}\right)=\mathcal{V}_{w}\left(P a_{1}\right)=\cdots=\mathcal{V}_{w}\left(P a_{n}\right)$. And thus, any such world tolerantly satisfies $P a_{n}$-indeed, strictly satisfies it.

But although $\operatorname{Prt}$ validates $n$-fold tolerance, adding $\neg P a_{n}$ to the premises of this argument results in an invalid argument. There is only one fact in $T\left(P a_{0} \wedge a_{0} \sim P\right.$ $\left.a_{1} \wedge \cdots \wedge a_{n-1} \sim_{P} a_{n} \wedge \neg P a_{n}\right)$, but this time it is not consistent; that is, our similarity restrictions have ruled out any consistent world containing this fact. Indeed, no world meeting our similarity restrictions can strictly satisfy $P a_{0} \wedge a_{0} \sim_{P}$ $a_{1} \wedge \cdots \wedge a_{n-1} \sim_{P} a_{n} \wedge \neg P a_{n}$. It is still tolerantly satisfiable, however. Among the worlds in $P R A G\left(P a_{0} \wedge a_{0} \sim_{P} a_{1} \wedge \cdots \wedge a_{n-1} \sim_{P} a_{n} \wedge \neg P a_{n}\right)$ are worlds that tolerantly satisfy every conjunct, and strictly satisfy all conjuncts but two similarity facts $a_{i} \sim_{P} a_{i+1}$ and $a_{j} \sim_{P} a_{j+1}$, with $i<j .{ }^{26}$ But at such worlds, we can have $P a_{k}=1$ for $0 \leq k \leq i$, plus $P a_{k}=\frac{1}{2}$ for $i<k \leq j$, plus $P a_{k}=0$ for $j<k \leq n$. In particular, then, such worlds do not even tolerantly satisfy $P a_{n}$; they show that $P a_{0}, a_{0} \sim_{P} a_{1}, \ldots a_{n-1} \sim_{P} a_{n}, \neg P a_{n} \not \nvdash^{P r t} P a_{n}$, although as we have seen the same argument without its final premise remains $\operatorname{Pr} t$-valid.

Is Prt transitive? Answering this question requires fixing on a sense of transitivity. As many usual senses of the term are not appropriate for nonmonotonic logics, this requires a bit of care, since as we have seen Prt is nonmonotonic. We will focus on a property like the one called Cut in, for example, Giordano et al. (2009, p. 18:6): If $\Gamma \vdash B$ and $\Gamma, B \vdash C$, then $\Gamma \vdash C .^{27}$ Does Prt have this property?

\footnotetext{
26 This assumes that $n>1$, to make room for distinct $i, j$. Where $n=1$, then, this exact example doesn't work. But one-step tolerance remains defeasible; it just takes a little more work to defeat it. In particular, $P a, a \sim_{P} b \vDash^{P r t} P b$ but $P a, a \sim_{P} b, \neg P a, \neg a \sim_{P} b \not \models P r t P b$.

27 Note that, without some appeal to monotonicity (and contraction), this is not equivalent to Cut as it occurs in Gentzen (1969).
} 
It does not. The core reason is that conclusions are held to a low standardtolerant satisfaction - while premises are pragmatically strengthened. And so we have $P a, \neg P a \vDash^{P r t} a \sim_{P} b$, plus $P a, \neg P a, a \sim_{P} b \vDash^{P r t} P b$; but $P a, \neg P a \not \models^{P r t} P b .^{28}$ The latter two claims are perhaps straightforward to see, but the first might be surprising. However, it does indeed hold. Any world $w$ in $P R A G(P a \wedge \neg P a)$ must be such that $\mathcal{V}_{w}(P a)=\frac{1}{2}$. As a result, no matter what $\mathcal{V}_{w}(P b)$ is, we have $\left|\mathcal{V}_{w}(P a)-\mathcal{V}_{w}(P b)\right|<1$, and so $1-\left|\mathcal{V}_{w}(P a)-\mathcal{V}_{w}(P b)\right|>0$. By our constraint, this requires $\mathcal{V}_{w}\left(a \sim_{P} b\right)>0$, and so $w$ tolerantly satisfies $a \sim_{P} b$.

\subsubsection{Pragmatic-to-pragmatic entailment $(\mathrm{Pr} P r)$}

There are reasons you might be unhappy with $\vDash^{P r t}$. We argued in Sects. 1 and 2 that nontransitivity and nonmonotonicity might be ways to account for vagueness. One might wonder, however, whether we need both of these properties. If either one suffices on its own to capture the situation, and if both are somehow seen as drawbacks, then we might hope for a purely nonmonotonic approach, just as st provides a purely nontransitive one. Second, if pragmatic interpretation captures what is meant by the speaker, one might wonder whether either conjunct can be inferred from the premise $P a \wedge \neg P a$. With this sentence the speaker wants to impart that $a$ is borderline tall. But if a conjunct like ' $P a$ ' is asserted alone, it is pragmatically interpreted to mean that $a$ is strictly tall, and thus that $a$ is not borderline tall. If we want a consequence relation capturing what can be asserted on the basis of antecedent assertions, the inference from $P a \wedge \neg P a$ to $P a$ should not be valid according to such a relation.

Indeed, experimental investigations of borderline cases point to participant willingness to agree with sentences of the form $P a \wedge \neg P a$ while disagreeing with $P a$ and $\neg P a$ individually (see Alxatib and Pelletier 2011; Égré et al. 2013). However, one important thing you might think a consequence relation ought to do is to constrain patterns of agreement and disagreement in the following way: if $\Gamma \vdash A$, then one ought not agree with everything in $\Gamma$ while disagreeing with $A .^{29}$ But then, if the participants in these experiments are not mistaken, indeed $P a \wedge \neg P a$ does not entail $P a$ or $\neg P a$, in any sense of entailment that bears this relation to their actions. What might such an entailment relation look like? Not Prt, since $P a \wedge \neg P a$ entails both $P a$ and $\neg P a$ in Prt.

To account for the latter type of consequence relation we therefore define the following inference relation (from pragmatic to pragmatic interpretation), again restricting ourselves to finite sets of premises, and stipulating that $P R A G(\bigwedge \emptyset)=$ $W$ :

\footnotetext{
28 The question of Prt's transitivity was settled by Shawn Standefer, who found this example. The corresponding question arose in footnote 22 of Cobreros et al. (2015a), but was mishandled; the reasoning we give there does not support the claim we make there. The tolerance constraint we give in that paper (in footnote 15) is different from the one we consider here, and we do not know whether the resulting consequence relation in that paper is transitive or not.

${ }^{29}$ For discussion of this point and related ideas, see MacFarlane (2004), Restall (2005), Ripley (2013) and Field (2015).
} 


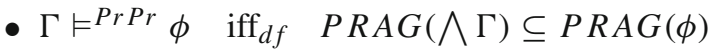

Thus, for inference we take into account what is (pragmatically) meant both by the premises and by the conclusion. It follows that $\models^{\operatorname{Pr} P r}$ does not satisfy conjunction elimination: in particular, $p \wedge \neg p \not \models P r P r \quad p$. Because $p \wedge \neg p$ will have no models where $p$ is strictly true, the pragmatic interpretation of the premise can only select models where $p$ is tolerantly true. Obviously, $p$ cannot be strictly true here: $p \wedge \neg p \not \models \operatorname{Pr} P r p$. However, even though $\phi \wedge \psi \not \nvdash^{\operatorname{Pr} P r} \phi$ in general, still $p \wedge q \models^{\operatorname{Pr} \operatorname{Pr}} p$. The reason is that $p$ and $q$ are independent of each other, and so there are models where $p \wedge q$ will receive value 1 ; these will be selected for the interpretation of the premise. In all such models, $p$ will indeed be strictly true.

As explained in Sect. 3.2.2, the pragmatic interpretation of a sentence of the form $(p \wedge \neg p) \vee q, P R A G((p \wedge \neg p) \vee q)$, will contain not only worlds where $q$ has value 1 , but also ones where $p$ is tolerantly true and $q$ is false. For this rea-

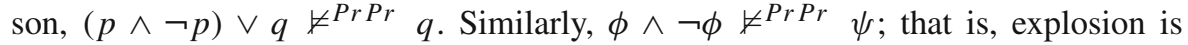
not valid. $\operatorname{Pr} P r$-entailment is again a paraconsistent entailment relation, just like Prt.

The tolerance inference, $P a, a \sim_{P} b \models^{\operatorname{Pr} P r} P b$, remains valid. Since the premises can be jointly strictly satisfied by a world, every $w \in P R A G\left(P a \wedge a \sim_{P} b\right)$ does indeed strictly satisfy them both, and so strictly satisfy $P b$ as well. Indeed, for essentially the same reasons as in $\operatorname{Prt}, n$-fold tolerance is $\operatorname{Pr} P r$-valid. (Note that the reasoning given to show this for Prt establishes the strict satisfaction of the conclusion of $n$-fold tolerance, assuming the pragmatic satisfaction of the premises.) However, in contrast to Prt, the conditional form of tolerance, $\left(P a \wedge a \sim_{P} b\right) \rightarrow P b$, is not a $\operatorname{Pr} P r$ theorem. To be a theorem, it would have to follow from the empty set; that is, $P R A G\left(\left(P a \wedge a \sim_{P} b\right) \rightarrow P b\right)$ would have to be $W$. But this is not so. ${ }^{30}$ This reflects the fact that, while tolerance conditionals are always at least tolerantly satisfied, asserting them need not be idle; their pragmatic strengthening still rules out some worlds.

Like $\operatorname{Prt}, \operatorname{Pr} \operatorname{Pr}$ too is nonmonotonic. Some examples of this nonmonotonicity were already present in Prt: $P a, a \sim_{P} b \vDash^{P r P r} P b$, but $P a, a \sim_{P} b, \neg P a, \neg a \sim_{P}$ $b \not \models \operatorname{PrPr} P b$. Others are specific to $\operatorname{Pr} \operatorname{Pr}$ : while $p \models^{\operatorname{Pr} P r} p$, still $p, \neg p \not \models \operatorname{Pr} \operatorname{Pr}$ $p$.

Unlike $\operatorname{Prt}$, however, $\operatorname{Pr} \operatorname{Pr}$ is transitive in a sense appropriate for nonmonotonic consequence relations: if $\Gamma \vDash^{\operatorname{Pr} P r} B$ and $\Gamma, B \vDash^{\operatorname{Pr} P r} C$, then $\Gamma \vDash \operatorname{Pr} \operatorname{Pr} C$. This follows from the fact that if $P R A G(\bigwedge \Gamma) \subseteq P R A G(B)$, then $P R A G(\bigwedge \Gamma) \subseteq$

\footnotetext{
30 Consider a world $w$ that gives us $\mathcal{V}_{w}(P \underline{d})=\frac{1}{2}$, and $\mathcal{V}_{w}\left(a \sim_{P} \underline{d}\right)=1$, for each $d \in D$. This world thinks everything is $P$-similar to $a$, that nothing isn't, and that everything both is and isn't $P$. Such a world is not in $P R A G\left(\left(P a \wedge a \sim_{P} b\right) \rightarrow P b\right)$. For suppose it was. Then there is some $f \in T\left(\left(P a \wedge a \sim_{P} b\right) \rightarrow P b\right)$ with $f \subseteq w$, and such that there is no $v<_{f} w$. Since $T\left(\left(P a \wedge a \sim_{P} b\right) \rightarrow P b\right)=\{\{\overline{\mathbf{P a}}\},\{\overline{\mathbf{a} \sim \mathbf{P} \mathbf{b}}\},\{\mathbf{P b}\}\}$, there are only three possible $f$ s, and $w$ cannot also contain $\left\{\overline{\mathbf{a} \sim_{\mathbf{P}} \mathbf{b}}\right\}$, so we can forget this one. This leaves $\{\overline{\mathbf{P a}}\}$ and $\{\mathbf{P b}\}$. However, whichever of these we consider for $f$, there is a $v<_{f} w$ : in the case of $\{\overline{\mathbf{P a}}\}$ it is the world that assigns the value 0 to every $P \underline{d}$, and in the case of $\{\mathbf{P b}\}$ it is the world that assigns the value 1 to each such. As a result, $w \notin P R A G\left(\left(P a \wedge a \sim_{P} b\right) \rightarrow P b\right)$.
} 
$P R A G(\bigwedge \Gamma \wedge B) .{ }^{31}$ So if $P R A G(\bigwedge \Gamma \wedge B) \subseteq P R A G(C)$, then $P R A G(\bigwedge \Gamma) \subseteq$ $P R A G(C)$ too.

\section{Sorites arguments}

With the machinery introduced in Sect. 3 we have come to define three different consequence relations, which we restrict to finite sets of premises:

$$
\begin{aligned}
\Gamma \vDash^{s t} \phi & \text { just in case there is no model } \mathcal{M} \text { such that, } \\
& \mathcal{V}_{\mathcal{M}}(\psi)=1 \text { for all } \psi \text { in } \Gamma \text { and } \mathcal{V}_{\mathcal{M}}(\phi)=0 . \\
\Gamma \vDash^{P r t} \phi & \text { just in case there is no model } \mathcal{M} \text { such that, } \\
& \mathcal{M} \in P R A G(\bigwedge \Gamma) \text { and } \mathcal{V}_{\mathcal{M}}(\phi)=0 . \\
\Gamma \models^{\operatorname{Pr} P r} \phi \quad & \text { just in case there is no model } \mathcal{M} \text { such that, } \\
& \mathcal{M} \in P R A G(\bigwedge \Gamma) \text { for all } \psi \text { in } \Gamma \text { and } \mathcal{M} \notin P R A G(\phi) .
\end{aligned}
$$

In Sect. 3 we presented some ways to capture similarity relations, in order to be able to express tolerance. In these logics tolerance is internalized since, in fact, the inference from 'Red(a)' and ' $a \sim_{\text {red }} b$ ' to 'Red(b)' is valid in each case. In this section we review how these logics deal with sorites arguments.

\subsection{The logics, side by side}

\subsection{1 st}

The logic st is based on the idea that premises and conclusions of an argument need not be subject to equal standards of satisfaction. If the premises of an argument are true to some strict standard, it suffices for validity if the conclusion is true to some less strict standard. Intuitively, this will lead to breaches of transitivity and this is precisely what happens, according to this logic, in sorites arguments. Since pragmatic satisfaction is not involved, st remains fully monotonic.

In our presentation of the sorites, we can formulate the argument as a step-by-step argument over the similarity relation or as an argument making use of tolerance as an explicit premise. According to $s t$ all steps in the sorites argument are valid. For example, for any two colored objects $a$ and $b$ that differ imperceptibly in hue within a more extended series, we have:

$$
\operatorname{Red}(\mathrm{a}), \mathrm{a} \sim \sim_{\operatorname{Red}} \mathrm{b} \vDash^{s t} \operatorname{Red}(\mathrm{b}),
$$

31 Let $A$ be $\wedge \Gamma$, and suppose $P R A G(A) \subseteq P R A G(B)$ and $w \in P R A G(A)$, to show $w \in P R A G(A \wedge B)$. Since $w \in P R A G(A)$, there is some $f \in T(A)$ with $f \subseteq w$ and no $v$ with $v<_{f} w$ and $f \subseteq v$. Similarly, since $w \in P R A G(B)$, there is some $g \in T(B)$ with $g \subseteq w$ and no $u$ with $u<g w$ and $g \subseteq u$. Since $T(A \wedge B)=T(A) \otimes T(B)$, we have $f \cup g \in T(A \wedge B)$.

If we can show, then, that there is no world $x$ with $x<f \cup g$ and $f \cup g \subseteq x$, then we have $w \in$ $P R A G(A \wedge B)$ and we're done. So suppose there is such an $x$. Since $x<_{f \cup g} w$, there must be some state of affairs $s$ in $f \cup g$ with $\bar{s} \in w$ but $\bar{s} \notin x$. s must be in either $f$ or $g$; wlog, suppose it is in $f$. But then $x<_{f} w$; and since $f \cup g \subseteq x$, we have also that $f \subseteq x$. Since $w \in P R A G(A)$, we know there is no such $x$. 
But it doesn't follow that the last element of the series is red, since when we put these arguments together, validity breaks; $n$-fold tolerance is not $s t$-valid. That is,

$$
\begin{gathered}
\operatorname{Red}\left(\mathrm{a}_{1}\right), \mathrm{a}_{1} \sim_{\operatorname{Red}} \mathrm{a}_{2} \models^{s t} \operatorname{Red}\left(\mathrm{a}_{2}\right) \quad \ldots \operatorname{Red}\left(\mathrm{a}_{\mathrm{n}-1}\right), \mathrm{a}_{\mathrm{n}-1} \sim_{\operatorname{Red}} \mathrm{a}_{\mathrm{n}} \models^{s t} \operatorname{Red}\left(\mathrm{a}_{\mathrm{n}}\right) \\
\operatorname{BUT} \\
\operatorname{Red}\left(\mathrm{a}_{1}\right), \mathrm{a}_{1} \sim_{\operatorname{Red}} \mathrm{a}_{2}, \ldots, \mathrm{a}_{\mathrm{n}-1} \sim_{\operatorname{Red}} \mathrm{a}_{\mathrm{n}} \not \nvdash^{s t} \operatorname{Red}\left(\mathrm{a}_{\mathrm{n}}\right)
\end{gathered}
$$

Concerning the tolerance principle,

$$
\forall \mathrm{x} \forall \mathrm{y}\left(\left(\operatorname{Red}(\mathrm{x}) \wedge \mathrm{x} \sim_{\operatorname{Red}} \mathrm{y}\right) \rightarrow \operatorname{Red}(\mathrm{y})\right)
$$

appropriate constraints on similarity render the principle $s t$-valid.

However, st does validate

$$
\begin{gathered}
\forall x \forall y\left(\left(\operatorname{Red}(x) \wedge x \sim_{\operatorname{Red}} y\right) \rightarrow \operatorname{Red}(y)\right), \operatorname{Red}\left(a_{1}\right), a_{1} \sim_{\operatorname{Red}} a_{2}, \ldots, \\
a_{n-1} \sim_{\operatorname{Red}} a_{n} \models^{s t} \operatorname{Red}\left(a_{n}\right)
\end{gathered}
$$

That is, although $n$-fold tolerance is not $s t$-valid, and although tolerance is already an $s t$-theorem, adding tolerance as a premise to $n$-fold tolerance results in a valid argument.

\subsubsection{Prt}

The logic Prt combines two features: pragmatic interpretation for the premises and tolerance for the conclusion. This results in a logic that is both nonmonotonic and nontransitive, as we've seen. Regarding step-by-step sorites arguments, Prt predicts all steps in the sorites argument to be valid. So for any element $a$ in the series,

$$
\operatorname{Red}(\mathrm{a}), \mathrm{a} \sim_{\operatorname{Red}} \mathrm{b} \vDash^{P r t} \operatorname{Red}(\mathrm{b})
$$

Unlike $s t, \operatorname{Prt}$ validates $n$-fold tolerance:

$$
\operatorname{Red}\left(a_{1}\right), a_{1} \sim_{\operatorname{Red}} a_{2}, \ldots, a_{n-1} \sim_{\operatorname{Red}} a_{n} \vDash^{P r t} \operatorname{Red}\left(a_{n}\right)
$$

But this cannot be arrived at by chaining the smaller inferences together; as we've seen, Prt is not in general transitive, and does not allow such chaining.

Moreover, Prt's nonmonotonicity means that similarity inferences can cease to hold in the presence of countervailing information. For example, we may have:

$$
\begin{gathered}
\operatorname{Red}(\mathrm{a}), \mathrm{a} \sim_{\operatorname{Red}} \mathrm{b} \models^{P r t} \operatorname{Red}(\mathrm{b}) \\
\operatorname{BUT} \\
\operatorname{Red}(\mathrm{a}), \mathrm{a} \sim_{\operatorname{Red}} \mathrm{b}, \neg \operatorname{Red}(\mathrm{a}), \neg \mathrm{a} \sim_{\operatorname{Red}} \mathrm{b} \not \nvdash^{P r t} \operatorname{Red}(\mathrm{b})
\end{gathered}
$$

Concerning the tolerance principle,

$$
\forall \mathrm{x} \forall \mathrm{y}((\operatorname{Red}(\mathrm{x}) \wedge \mathrm{x} \sim \operatorname{Red} \mathrm{y}) \rightarrow \operatorname{Red}(\mathrm{y}))
$$


as in the case of $s t$, is Prt-valid. Unlike in $s t$, however, adding this tolerance sentence as a premise to an $n$-fold tolerance argument makes no difference: the argument is valid both with and without such a tolerance premise.

\subsection{3 $\operatorname{Pr} P r$}

The driving idea for $\operatorname{Pr} P r$ is that the validity of an argument should be evaluated in connection with those models that provide the highest standards of satisfaction compatible with the statements contained in the premises and in the conclusions. Just as with our other logics, according to $\operatorname{Pr} P r$ all steps in the sorites argument are valid. So for any element $a$ in the series,

$$
\operatorname{Red}(\mathrm{a}), \mathrm{a} \sim_{\operatorname{Red}} \mathrm{b} \vDash^{\operatorname{Pr} P r} \operatorname{Red}(\mathrm{b})
$$

As we've seen, in contrast to either st or $\operatorname{Prt}, \operatorname{Pr} \operatorname{Pr}$ is transitive and, therefore, validity won't break down when we put these arguments together. That is, since for each $j \leq n$ we have

$$
\operatorname{Red}\left(a_{1}\right), \ldots, \operatorname{Red}\left(a_{j-1}\right), a_{1} \sim_{\operatorname{Red}} a_{2}, \ldots, a_{j-1} \sim_{\operatorname{Red}} a_{j} \models^{\operatorname{Pr} P r} \operatorname{Red}\left(a_{j}\right)
$$

we can put these together by repeated appeals to transitivity to arrive at

$$
\operatorname{Red}\left(a_{1}\right), a_{1} \sim_{\operatorname{Red}} a_{2}, \ldots, a_{n-1} \sim_{\operatorname{Red}} a_{n} \vDash^{\operatorname{Pr} P r} \operatorname{Red}\left(a_{n}\right)
$$

Again, nonmonotonicity allows us to render the inference invalid by adding countervailing information in the premises:

$$
\operatorname{Red}\left(a_{1}\right), a_{1} \sim_{\operatorname{Red}} a_{2}, \ldots, a_{n-1} \sim_{\operatorname{Red}} a_{n}, \neg \operatorname{Red}\left(a_{n}\right) \not{ }^{\operatorname{Pr} P r} \operatorname{Red}\left(a_{n}\right)
$$

Unlike in st and Prt, tolerance sentences are not theorems in $\operatorname{Pr} P r$. However, like $\operatorname{Prt}$ (and unlike $s t$ ), tolerance sentences make no difference for validity when they are added as premises to $n$-fold tolerance: with or without additional tolerance premises, $n$-fold tolerance is $\operatorname{Pr} P r$-valid.

\subsection{Conclusion}

In the course of this paper, we have outlined three distinct consequence relations: $s t$, Prt, and $\operatorname{Pr} P r$. Of these, the first is monotonic but nontransitive, the second is both nonmonotonic and nontransitive, and the third is nonmonotonic but transitive. They have much in common: all validate the tolerance inference, and each allows the soft status of tolerance to be recognised by failing to obey the full budget of usual structural rules.

Each relation reveals a different aspect of the underlying space of models, and of the way those models operate in pragmatic strengthening. These models draw together 
tools from many-valued and nonmonotonic logics to generate appropriate predictions about what is assertible, even in the kinds of tricky cases we've discussed.

Since we have defined three kinds of satisfaction (strict, tolerant, pragmatic), we have the raw materials to define nine different two-sided consequence relations, of which we have explored three in a bit of detail. None of these is itself any full story; rather they all reveal different interactions between strict satisfaction, tolerant satisfaction, and the pragmatic processes we have outlined. Overall, then, this paper's approach not only explains how tolerance can be valid without the sorites wreaking disaster, it also gives a detailed picture of the various ways in which tolerance is valid.

Open Access This article is distributed under the terms of the Creative Commons Attribution 4.0 International License (http://creativecommons.org/licenses/by/4.0/), which permits unrestricted use, distribution, and reproduction in any medium, provided you give appropriate credit to the original author(s) and the source, provide a link to the Creative Commons license, and indicate if changes were made.

\section{References}

Alxatib, S., Pagin, P., \& Sauerland, U. (2013). Acceptable contradictions: Pragmatics or semantics? A reply to Cobreros, et al. Journal of Philosophical Logic, 42(4), 619-634.

Alxatib, S., \& Pelletier, J. (2011). The psychology of vagueness: Borderline cases and contradictions. Mind and Language, 26(3), 287-326.

Asher, N. (1992). A default, truth conditional semantics for the progressive. Linguistics and philosophy, 15, 463-508.

Asher, N., \& Lascarides, A. (2003). Logics of conversation. Cambridge: Cambridge University Press.

Asher, N., \& Morreau, M. (1991). Commonsense entailment: A modal theory of nonmonotonic reasoning. In J. van Eijck (Ed.), Logics in AI. JELIA 1990. Lecture notes in Computer Science (Lecture notes in Artificial Intelligence) (Vol. 478). Berlin: Springer.

Asher, N., \& Morreau, M. (1995). What some generic sentences mean. In G. Carlson \& F . J. Pelletier (Eds.), The generic book (pp. 300-330). Chicago: University of Chicago Press.

Bennett, B. (2006). A theory of vague adjectives grounded in relevant observables. In P. Doherty, J. Mylopoulos, \& C. Welty (Eds.), Proceedings of the tenth international conference on principles of knowledge representation and reasoning (pp. 36-45). Palo Alto: AAAI Press.

Bimbó, K. (2015). Proof theory: Sequent calculi and related formalisms. Boca Raton, FL: CRC Press.

Burnett, H. (2014). A delineation solution to the puzzles of absolute adjectives. Linguistics and Philosophy, 37(1), 1-39.

Cobreros, P., Egré, P., Ripley, D., \& van Rooij, R. (2012). Tolerant, classical, strict. Journal of Philosophical Logic, 41(2), 347-385.

Cobreros, P., Égré, P., Ripley, D., \& Van Rooij, R. (2013). Reaching transparent truth. Mind, 122(488), 841-866.

Cobreros, P., Égré, P., Ripley, D., \& van Rooij, R. (2015a). Pragmatic interpretations of vague expressions: Strongest meaning and nonmonotonic consequence. Journal of Philosophical Logic, 44(4), 375-393.

Cobreros, P., Egré, P., Ripley, D., \& van Rooij, R. (2015b). Vagueness, truth and permissive consequence. In T. Achourioti, H. Galinon, K. Fujimoto, \& J. Martínez-Fernández (Eds.), Unifying the philosophy of truth (pp. 409-430). Springer.

Cobreros, P., Egré, P., Ripley, D., \& van Rooij, R. (2017). Tolerance and degrees of truth (manuscript).

Cumming, S. (2014). Applying semantic conventions to cases: A default-based account. In Talk given at the 2014 Phlip conference, Tarrytown.

Edgington, D. (1997). Vagueness by degrees. In R. Keefe \& P. Smith (Eds.), Vagueness: A reader (pp. 294-316). Cambridge, MA: MIT Press.

Égré, P. (2015). Vagueness: Why do we believe in tolerance? Journal of Philosophical Logic, 44(6), 663679.

Égré, P., de Gardelle, V., \& Ripley, D. (2013). Vagueness and order effects in color categorization. Journal of Logic, Language, and Information, 22(4), 391-420. 
Égré, P., Ripley, D., \& Verheyen, S. (2017). The sorites paradox in psychology. In E. Zardini \& S. Oms (Eds.), The sorites paradox. Cambridge: Cambridge University Press.

Egré, P., \& Zehr, J. (2016). Are gaps preferred to gluts? a closer look at borderline contradictions. In E. Castroviejo, G. W. Sassoon, \& L. McNally (Eds.), The semantics of gradability, vagueness, and scale structure-Experimental perspectives. New York: Springer. (forthcoming) .

Field, H. (2015). What is logical validity? In C. Caret \& O. Hjortland (Eds.), Foundations of logical consequence (pp. 33-70). Oxford: Oxford University Press.

Fine, K. (2014). Truth-maker semantics for intuitionistic logic. Journal of Philosophical Logic, 43, 549-577.

Frankowski, S. (2004). Formalization of a plausible inference. Bulletin of the Section of Logic, 33, 41-52.

Gentzen, G. (1969). Investigations into logical deduction. In M. E. Szabo (Ed.), The collected papers of Gerhard Gentzen (pp. 68-131). Amsterdam: North-Holland Publishing Company.

Giordano, L., Gliozzi, V., Olivetti, N., \& Pozzato, G. L. (2009). Analytic tableaux calculi for KLM logics of nonmonotonic reasoning. ACM Transactions on Computational Logic, 10(3), 18:1-18:47.

Goguen, J. A. (1969). The logic of inexact concepts. Synthese, 19(3), 325-373.

Grice, H. P. (1967). Logic and conversation, typescript from the William James Lectures, Harvard University. (Published in Studies in the Way of Worlds, pp. 22-40, by P. Grice, 1989, Cambridge, MA: Harvard University Press).

Hallnäs, L. (1991). Partial inductive definitions. Theoretical Computer Science, 87(1), 115-142.

Hallnäs, L., \& Schroeder-Heister, P. (1991). A proof-theoretic approach to logic programming II: Programs as definitions. Journal of Logic and Computation, 1(5), 635-660.

Hu, I. (2015). A generic theory of vagueness (manuscript).

Kamp, H. (1981). The paradox of the heap. Aspects of philosophical logic. Dordrecht: D. Reidel.

Kennedy, C., \& McNally, L. (2005). Scale structure and the semantic typology of gradable predicates. Language, 81(2), 345-381.

Kratzer, A. (2016). Situations in natural language semantics. In Zalta, E. N., editor, The Stanford Encyclopedia of Philosophy. Metaphysics Research Lab, Stanford University, spring 2016 edition.

Lakoff, G. (1973). Hedges: A study in meaning criteria and the logic of fuzzy concepts. Journal of Philosophical Logic, 2(4), 458-508.

MacFarlane, J. (2004). In what sense (if any) is logic normative for thought? (manuscript).

Machina, K. (1976). Truth, belief and vagueness. Journal of Philosophical Logic, 5, 47-78.

Maudlin, T. (2008). Grading, sorting, and the sorites. Midwest Studies in Philosophy, 32(1), 141-168.

McCarthy, J. (1980). Circumscription-A form of non-monotonic reasoning. Artificial Intelligence, 13, 27-39.

McCready, E. (2012). Emotive equilibria. Linguistics and Philosophy, 35, 243-283.

Meyer, R. K., \& McRobbie, M. A. (1982a). Multisets and relevant implication I. Australasian Journal of Philosophy, 60(2), 107-139.

Meyer, R. K., \& McRobbie, M. A. (1982b). Multisets and relevant implication II. Australasian Journal of Philosophy, 60(3), 265-281.

Millikan, R. G. (1984). Language, thought, and other biological categories. Cambridge, MA: MIT Press.

Misiuna, K. (2010). A certain consequence relation for solving paradoxes of vagueness. Logique et Analyse, 53(209), 25-50.

Paoli, F. (2002). Substructural logics: A primer. Dordrecht: Kluwer Academic Publishing.

Paoli, F. (2003). A really fuzzy approach to the sorites paradox. Synthese, 134(3), 363-387.

Priest, G. (1991). Minimally inconsistent lp. Studia Logica, 50(2), 321-331.

Ray, K. S., \& Chakraborty, A. (2011). A fuzzy version of default logic. International Journal of Intelligent Computing and Cybernetics, 4(1), 5-24.

Restall, G. (2000). An introduction to substructural logics. London: Routledge.

Restall, G. (2005). Multiple conclusions. In P. Hájek, L. Valdés-Villanueva, \& D. Westerståhl (Eds.), Logic, methodology, and philosophy of science: Proceedings of the twelfth international congress (pp. 189205). London: Kings' College Publications.

Ripley, D. (2011). Contradictions at the borders. In R. Nouwen, R. van Rooij, H.-C. Schmitz, \& U. Sauerland (Eds.), Vagueness and communication (pp. 169-188). New York: Springer.

Ripley, D. (2013). Paradoxes and failures of cut. Australasian Journal of Philosophy, 91(1), 139-164.

Ripley, D. (2017). On the 'transitivity' of consequence relations. Journal of Logic and Computation (forthcoming).

Routley, R., Meyer, R. K., Plumwood, V., \& Brady, R. T. (1982). Relevant logics and their rivals 1. Atascadero, CA: Ridgeview. 
Schulz, K., \& Van Rooij, R. (2006). Pragmatic meaning and non-monotonic reasoning: The case of exhaustive interpretation. Linguistics and Philosophy, 29(2), 205-250.

Serchuk, P., Hargreaves, I., \& Zach, R. (2011). Vagueness, logic, and use: Four experimental studies on vagueness. Mind and Language, 26(5), 540-573.

Slaney, J. K. (2010). A logic for vagueness. Australasian Journal of Logic, 8:100-134. Adapted from technical report TR-ARP-15-1988, Australian National University.

Smith, N . J. (2008). Vagueness and degrees of truth. Oxford: Oxford University Press.

Tennant, N. (1987). Anti-realism and logic. Oxford: Oxford University Press.

Thomason, R. (2011). Non-monotonicity in linguistics, volume handbook of logic and language (2nd ed., pp. 781-837). Amsterdam: Elsevier.

van Fraassen, B. C. (1969). Facts and tautological entailments. The Journal of Philosophy, 66(15), 477-487.

van Rooij, R. (2011a). Revealed preference and satisficing behavior. Synthese, 179(1), 1-12.

van Rooij, R. (2011b). Vagueness and linguistics. In Vagueness: A guide (pp. 123-170). Springer.

van Rooij, R. (2017). A fine-grained global analysis of implicatures. In S. Pistoia-Reda \& F. Domaneschi (Eds.), Linguistic and psycholinguistic approaches to implicatures and presuppositions. Palgrave Macmillan: Palgrave Macmillan.

van Rooij, R., \& Schulz, K. (2011). Non-monotonic reasoning in interpretation-Recent developments. In J. van Benthem \& A. ter Meulen (Eds.), Handbook of Logic and Language (2nd ed., pp. 781-837). Amsterdam: Elsevier.

Weir, A. (1998). Naive set theory, paraconsistency, and indeterminacy: Part I. Logique et Analyse, 41(161163), 219-266.

Williamson, T. (1994). Vagueness. London: Routledge.

Wright, C. (1975). On the coherence of vague predicates. Synthese, 30, 325-365.

Zardini, E. (2008). A model of tolerance. Studia Logica, 90(3), 337-368.

Zhang, J., Ding, Y., and Gong, J. (2003). Intrusion detection system based on fuzzy default logic. In FUZZ'03. The 12th IEEE International Conference on Fuzzy Systems (Vol. 2, pp. 1350-1356). IEEE. 